\title{
The Additive Effects of Core Muscle Strengthening and Trunk NMES on Trunk Balance in Stroke Patients
}

\author{
Eun Jae Ko, $\mathrm{MD}^{1}$, Min Ho Chun, $\mathrm{MD}, \mathrm{PhD}^{1}$, Dae Yul Kim, $\mathrm{MD}, \mathrm{PhD}^{1}$, \\ Jin Hwa Yi, $\mathrm{MD}^{2}$, Won Kim, $\mathrm{MD}^{1}$, Jayoung Hong, $\mathrm{MD}^{3}$ \\ ${ }^{1}$ Department of Rehabilitation Medicine, Asan Medical Center, University of Ulsan College of Medicine, Seoul; \\ ${ }^{2}$ Department of Physical Medicine and Rehabilitation Medicine, Dream Hospital, Seoul; \\ ${ }^{3}$ Department of Rehabilitation Medicine, Raphael Geriatric Hospital, Suwon, Korea
}

\begin{abstract}
Objective To investigate an additive effect of core muscle strengthening (CMS) and trunk neuromuscular electrical stimulation (tNEMS) on trunk balance in stroke patients

Methods Thirty patients with acute or subacute stroke who were unable to maintain static sitting balance for $>5$ minutes were enrolled and randomly assigned to 3 groups, i.e., patients in the CMS $(n=10)$ group received additional CMS program; the tNMES group $(n=10)$ received additional tNMES over the posterior back muscles; and the combination (CMS and tNMES) group $(n=10)$ received both treatments. Each additional treatment was performed 3 times per week for 20 minutes per day over 3 weeks. Korean version of Berg Balance Scale (K-BBS), total score of postural assessment scale for stroke patients (PASS), Trunk Impairment Scale (TIS), and Korean version of Modified Barthel Index (K-MBI) were evaluated before and after 3 weeks of therapeutic intervention.

Results All 3 groups showed improvements in K-BBS, PASS, TIS, and K-MBI after therapeutic interventions, with some differences. The combination group showed more improvements in K-BBS and the dynamic sitting balance of TIS, as compared to the CMS group; and more improvement in K-BBS, as compared to the tNMES group.

Conclusion The results indicated an additive effect of CMS and tNMES on the recovery of trunk balance in patients with acute or subacute stroke who have poor sitting balance. Simultaneous application of CMS and tNMES should be considered when designing a rehabilitation program to improve trunk balance in stroke patients.
\end{abstract}

Keywords Stroke, Electrical stimulation, Exercise

Received January 12, 2015; Accepted August 7, 2015

Corresponding author: Min Ho Chun

Department of Rehabilitation Medicine, Asan Medical Center, University of Ulsan College of Medicine, 88 Olympic-ro 43-gil, Songpa-gu, Seoul 05505, Korea

Tel: +82-2-3010-3800, Fax: +82-2-3010-6964, E-mail: mhchun@amc.seoul.kr

(ㄷ) This is an open-access article distributed under the terms of the Creative Commons Attribution Non-Commercial License (http://creativecommons.org/ licenses/by-nc/4.0) which permits unrestricted noncommercial use, distribution, and reproduction in any medium, provided the original work is properly cited. Copyright ( 2016 by Korean Academy of Rehabilitation Medicine 


\section{INTRODUCTION}

Stroke is a serious and disabling health problem that is common worldwide [1]. Following a stroke, patients frequently suffer severe disability and limitations in activities of daily living (ADL). Postural instability is one of the major morbidities following a stroke, which is associated with an increased risk of falling.

Postural control is the ability of the body to maintain an upright stance, which is stabilized by muscle in the hip and lower back, and is essential for performing both ADL and instrumental ADL (IADL) [2]. Trunk imbalance in hemiparetic stroke patients results from proprioceptive sense impairment or paralysis of the limb and trunk [3]. Trunk muscles are innervated by the bilateral cerebral hemisphere [4], therefore the function of the trunk muscles in hemiplegic patients is relatively conserved, as compared to the weaker limbs [5]. Thus, unilateral stroke could potentially result in functional deterioration of the trunk muscles on both the contralateral and ipsilateral sides of the body, although it affects patients to a lesser extent on the lesional side [6]. There are several reports of post-stroke trunk postural control defects in standing [7] and sitting [8].

Assessments of trunk control at an early stage after stroke have predictive value for comprehensive ADL function in patients who survive for 6 months and therefore is recommended [9]. Despite evidence demonstrating the importance of trunk performance after stroke, trunk muscle strengthening is often neglected and weak limbs is the focus of most reported studies $[10,11]$.

Research has shown that core muscle strengthening (CMS) is a beneficial therapeutic technique for the improvement of trunk balance in patients with acute, subacute or chronic hemiparetic stroke [5,12]. Furthermore, another study on trunk neuromuscular electrical stimulation (tNMES) concluded that it was effective in improving trunk control in patients with acute and subacute hemiparetic stroke [13].

In the present study, we aimed to investigate the additive effects of CMS and tNMES on trunk balance in stroke patients.

\section{MATERIALS AND METHODS}

\section{Study design and participants}

This cohort study was prospectively carried out at Asan Medical Center.

Patients who were admitted or transferred to the Department of Rehabilitation Medicine at Asan Medical Center because of acute or subacute stroke that included both infarction and hemorrhage, were included in the trial. Patients with a first stroke of hemiparesis within 1 month of onset, and who could not maintain static sitting balance for more than 5 minutes were enrolled. Exclusion criteria were as follows: 1 ) subjects who could not communicate with therapists as a consequence of severe aphasia or cognitive impairment, 2) subjects who were paralyzed on both sides, 3) subjects who were suffering from other neurological diseases, 4) subjects with neglect, 5) subjects with vestibular organ diseases, 6) subjects with severe internal diseases or back pain, and 7) subjects with implanted pacemakers or defibrillators.

Patients or a proxy provided written informed consent. Ethical approval was obtained from Asan Medical Center.

\section{Randomization}

Between June 2013 and November 2013, 34 patients were enrolled at Asan Medical Center. On the day of recruitment, patients were randomly assigned to 1 of 3 groups using a random table: the CMS group $(n=12)$, the tNMES group $(n=11)$, or the combination (CMS and tNMES) group ( $n=11)$. Four patients dropped out of the study for the following reasons: 2 were lost to follow-up because of early discharge before the study completion, and 2 could not participate in the rehabilitation program because of a poor medical condition (Fig. 1). A total of 30 patients completed the study and were included in the analysis. None of the patients had to stop the study due to tiredness or pain.

All therapists were randomly assigned to a patient; and all participating patients were aware of the treatment allocation in the study design. However, the 2 fixed therapists involved in patient evaluation but not treatment, and 3 investigators who conducted the study, were blinded to the treatment allocation.

\section{Interventions}

Patients in 3 groups participated in a standard rehabili- 


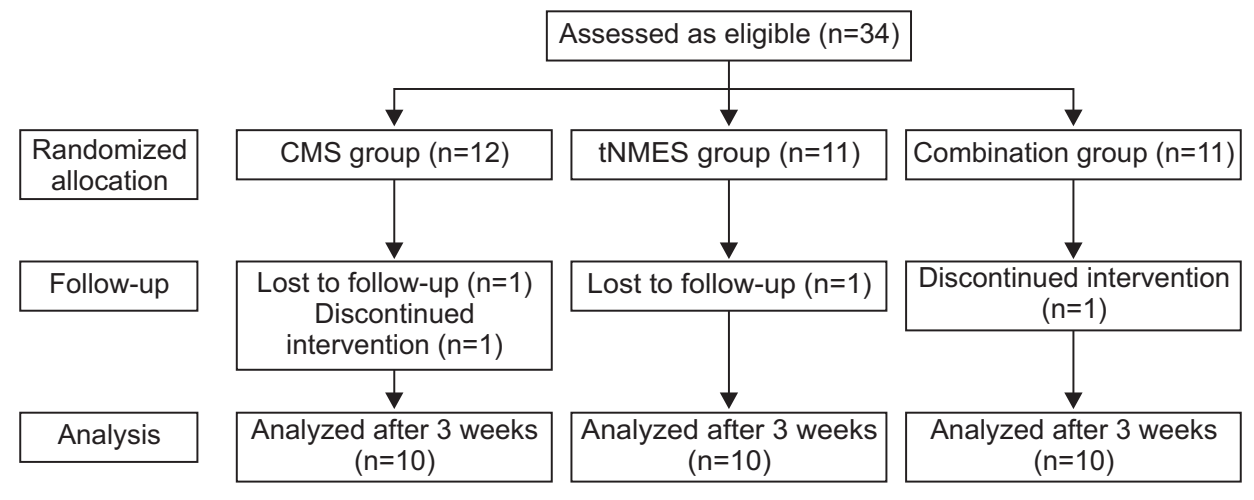

Fig. 1. Flowchart of the study. CMS group, core muscle strengthening group; tNMES group, trunk neuromuscular electrical stimulation group; Combination group, core muscle strengthening and trunk neuromuscular electrical stimulation group.

tation program consisting of physiotherapy and occupational therapy, including a range of movement exercises, tone facilitation, strengthening, balancing, ADL training, and ordinary postural control exercises, such as standing, shifting of weight loads between the nonparalytic and paralytic sides of the foot, and gait. Cognitive therapy and speech therapy were provided if needed. However, patients assigned to the CMS group received an additional CMS program, patients in the tNMES group received additional tNMES over the posterior back muscles, and patients in the combination group received CMS program with tNMES on their back. Each additional treatment was performed thrice weekly for 20 minutes per day over a period of 3 weeks. The total rehabilitation time was similar for all patients.

For the tNMES group, we used 4 channel electrodes, and attached them to the thoracic erector spinae $(5 \mathrm{~cm}$ lateral to the $\mathrm{T} 6$ spinous process), and lumbar erector spinae ( $2 \mathrm{~cm}$ lateral to the $\mathrm{L} 5$ spinous process) as shown in Fig. 2. Electrical stimulation (Novastim CU-FS1; CU Medical Systems Inc., Wonju, Korea) was applied at 30-70 $\mathrm{mA}$ intensity, $250 \mathrm{~ms}$ pulse width, and $35 \mathrm{~Hz}$ frequency for 10 seconds followed by a pause for 12 seconds. The intensity of stimulation was set to the maximum amount at which patients could feel muscle contraction without pain sensation or tiredness. Patients were told to maintain a sitting position as independently as possible.

The CMS program focused on trunk muscle strengthening, selective movements of the trunk muscle, and coordination, and was carried out in supine, prone, and lateral positions (Fig. 3). In a supine position, patients were told to bend their legs, rest their feet on the treatment table, keep their back in a neutral position and tighten their abdominal muscles. The exercises included lifting their pelvis with both feet supported (the bridge

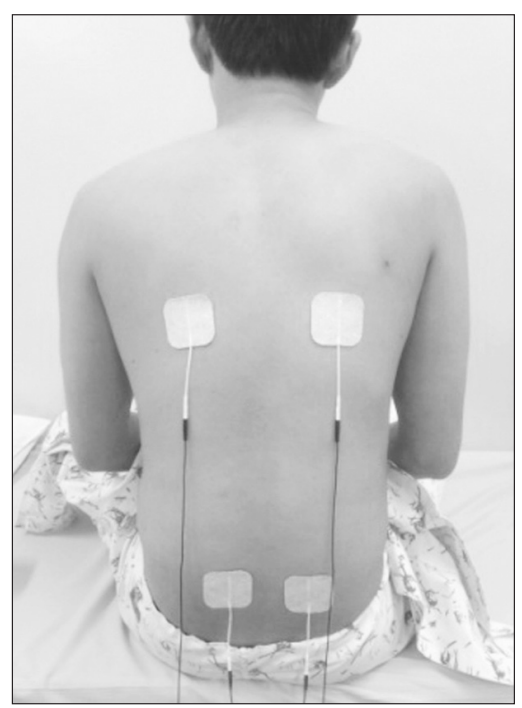

Fig. 2. Surface electodes were placed at 4 sites. Two were on the thoracic erector spinae, and the other 2 were on the lumbar erector spinae.

exercise), tilting both knees slowly to the left and right sides as far as possible (segmental rotation), and raising both legs and arms off the floor and towards the ceiling before lowering one arm and the opposite leg simultaneously and then repeating for the other side (the dead bug exercise). Prone position exercises included maintaining a push-up position with the body weight borne on the forearms, elbow, and toes (plank exercise), pushing hips up while keeping the back straight from a plank position and tightening the abdominal muscles (belly blaster), and kneeling on the floor with hands placed approximately shoulder width apart before lifting one hand and the opposite knee (bird dog exercise). In a lateral position, exercises consisted of lying on one side, balancing on a forearm and foot to form a diagonal line while maintaining shoulders, hips, and knees in alignment and 

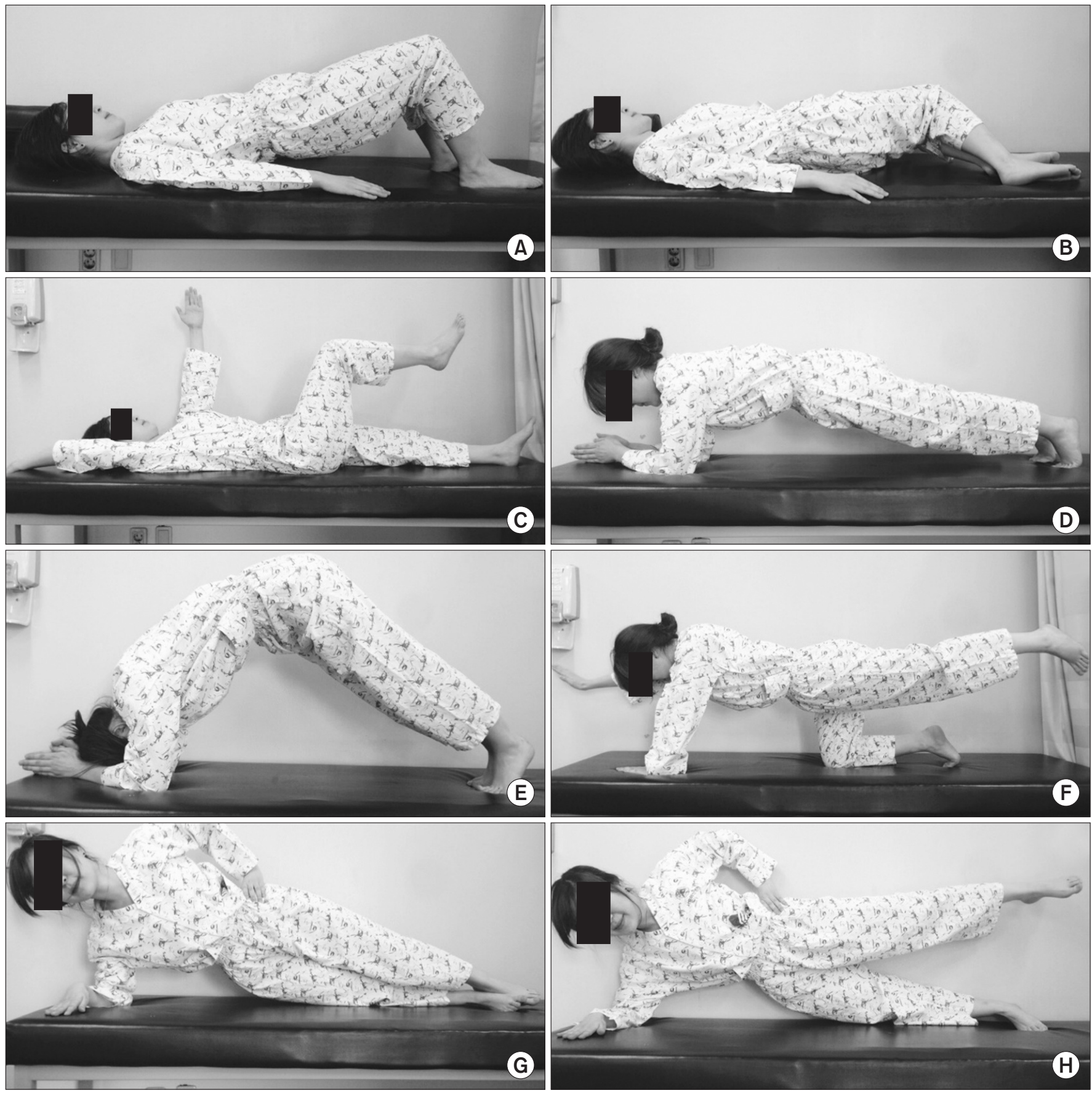

Fig. 3. The core muscle strengthening (CMS) program consisted of (A) the bridge exercise, (B) segmental rotation, (C) the dead bug exercise, (D) plank exercise, (E) belly blaster, (F) bird dog exercise, (G) side plank exercise, and (H) side bridge exercise.

tightening the abdominal muscles (side plank exercise), and lifting and lowering the opposite leg (side bridge exercise). Exercises were introduced gradually and the progression and number of repetitions were based on the level of performances for each patient. Therefore, some patients only repeated low level exercises, while some progressed to more difficult exercises. Trunk exercises were initiated with moderate assistance and progressed to a state requiring no assistance. Patients who did not have sufficient muscle strength to control themselves 
were assisted by their therapists during exercises.

Lastly, the patients in the combination group were told to receive the CMS program with tNMES while on their back.

\section{Patient assessments}

Patients were evaluated before, and after 3 weeks of a therapeutic intervention by both physical and occupational therapists. The primary outcome was assessed using the Korean version of Berg Balance Scale (K-BBS; scored as 0-56) [14], for trunk balance, and the secondary outcomes were scored using the following criteria: total score of Postural Assessment Scale for Stroke patients (PASS; scored as 0-36) [15] and Trunk Impairment Scale (TIS; scored as 0-23) [16] for trunk balance, and the Korean version of Modified Barthel Index (K-MBI; scored as 0-100) for ADL.

Gender, age, side of weakness, stroke etiology, time post stroke, and Korean version of the Mini-Mental Status Examination (K-MMSE) were investigated in each patient.

\section{Statistical analysis}

Data were analyzed using SPSS for Windows ver. 20.0 (IBM SPSS, Armonk, NY, USA) and the threshold for statistical significance was set to $p<0.05$. For baseline characteristics, we used the chi-square test or Fisher exact test for categorical variables, and the Kruskal-Wallis test for continuous variables. Mean, standard deviation, median, and interquartile range of data were obtained. The Wilcoxon signed-rank test was used for comparisons among the 3 groups before and after treatment. The KruskalWallis test was used to measure improvements after reha- bilitation, and the Mann-Whitney U test was used with a Bonferroni corrected, post hoc test at a threshold for statistical significance set of $p<0.025$. Spearman correlation analyses were used to examine the relationship between trunk balance and ADL.

\section{RESULTS}

\section{Baseline characteristics}

The CMS group included 8 males and 2 females, the tNMES group included 4 males and 6 females, and the combination group included 7 males and 3 females. Overall, there were more male than female subjects. The median ages were as follows: CMS group, 59.5 years; tNMES group, 65.5 years; and combination group, 58.5 years. The side of weakness was similar in all 3 groups. For the stroke etiology, 26 patients had a cerebral infarction and 4 patients had a cerebral hemorrhage. The number of enrolled patients with cerebral infarction was higher than those with cerebral hemorrhage in all 3 groups. The amount of post-stroke did not show any significant differences among the groups. Finally, patient baseline characteristics were well balanced among the 3 groups (Table 1).

\section{Effects before and after treatment}

Table 2 showed primary and secondary patient outcomes before and after treatment in each group. All 3 groups showed improvements in K-BBS, PASS, TIS, and K-MBI after therapeutic intervention, with some differences.

Table 1. Baseline characteristics of patients

\begin{tabular}{lccc}
\hline & CMS group (n=10) & tNMES group (n=10) & Combination group (n=10) \\
\hline Gender (male:female) & $8: 2$ & $4: 6$ & $7: 3$ \\
Age (yr) & $59.5(53.5-71.5)$ & $65.5(49.8-69)$ & $58.5(45.5-72.5)$ \\
Side of weakness (right:left) & $5: 5$ & $6: 4$ & $4: 6$ \\
Etiology (infarction:hemorrhage) & $8: 2$ & $9: 1$ & $9: 1$ \\
Time post stroke (day) & $12(8-14.3)$ & $8.5(8-13.3)$ & $11(8.5-13)$ \\
K-MMSE & $21.5(15-27.3)$ & $26.5(23.8-28.3)$ & $26.5(24.3-29)$ \\
\hline
\end{tabular}

Values are presented as number of patients or median (interquartile range).

CMS group, core muscle strengthening group; tNMES group, trunk neuromuscular electrical stimulation group; Combination group, core muscle strengthening and trunk neuromuscular electrical stimulation group; K-MMSE, Korean version of the Mini-Mental Status Examination. 
Table 2. K-BBS, PASS, TIS, and K-MBI before and after treatment in each group

\begin{tabular}{|c|c|c|c|c|c|c|}
\hline & \multicolumn{2}{|c|}{ CMS group $(n=10)$} & \multicolumn{2}{|c|}{ tNMES group $(n=10)$} & \multicolumn{2}{|c|}{ Combination group $(n=10)$} \\
\hline & Pre & Post & Pre & Post & Pre & Post \\
\hline \multirow[t]{2}{*}{ K-BBS } & $17.7 \pm 17.2$ & $28.4 \pm 17.6^{*}$ & $14.0 \pm 13.9$ & $27.4 \pm 16.3^{*}$ & $14.9 \pm 11.5$ & $40.0 \pm 11.7^{*}$ \\
\hline & $12.5(3-28.3)$ & $31(12.8-41.5)$ & $13.5(4-24.3)$ & $24.5(13-46.3)$ & $14(4.8-25.5)$ & $42.5(34-48.8)$ \\
\hline \multirow[t]{2}{*}{ PASS } & $19.7 \pm 10.3$ & $27.0 \pm 10.6^{*}$ & $15.2 \pm 10.0$ & $26.6 \pm 5.9^{*}$ & $17.9 \pm 9.0$ & $32.3 \pm 3.0^{*}$ \\
\hline & $20(13.5-27)$ & $31.5(20.5-35)$ & $15.5(8-22)$ & $27.5(23-32.3)$ & $17.5(11.5-26.3)$ & $33.5(30-34.3)$ \\
\hline \multirow[t]{2}{*}{ TIS } & $9.3 \pm 6.5$ & $14.0 \pm 7.4^{*}$ & $9.4 \pm 6.6$ & $13.2 \pm 6.0^{*}$ & $8.0 \pm 4.7$ & $15.1 \pm 4.0^{*}$ \\
\hline & $9(3-13.8)$ & $16.5(9.5-20)$ & $8(4.5-12.8)$ & $13.5(8.3-18.8)$ & $8.5(3-12.3)$ & $15.5(13.8-16.8)$ \\
\hline \multirow[t]{2}{*}{ K-MBI } & $29.8 \pm 22.0$ & $58.4 \pm 30.8^{*}$ & $31.7 \pm 21.8$ & $56.2 \pm 20.6^{*}$ & $43.0 \pm 20.2$ & $72.4 \pm 14.0^{*}$ \\
\hline & $32(15.5-45.8)$ & $68.5(45.5-77)$ & $31.5(19-48.3)$ & $52.5(39.8-67.8)$ & $43.5(23.3-56)$ & $72.5(60.3-86.8)$ \\
\hline
\end{tabular}

Values are presented as mean \pm standard deviation or median (interquartile range).

CMS group, core muscle strengthening group; tNMES group, trunk neuromuscular electrical stimulation group; Combination group, core muscle strengthening and trunk neuromuscular electrical stimulation group; K-BBS, Korean version of Berg Balance Scale; PASS, Total score of postural assessment scale for stroke patients; TIS, Trunk Impairment Scale; K-MBI, Korean version of Modified Barthel Index.

${ }^{*} \mathrm{p}<0.05$.

Comparisons of post-treatment effects in the three groups

For comparison of improvements after therapeutic intervention, K-BBS and the dynamic sitting balance of TIS in the combination group were significantly higher than in the other 2 groups. The post-hoc test indicated that the combination group showed more improvements in KBBS and the dynamic sitting balance of TIS, as compared to the CMS group; and more improvement in K-BBS, as compared to the tNMES group (Fig. 4). Changes in PASS, static sitting balance and the total score of TIS tended to be higher in the combination group, as compared to the other groups, but without statistical significance.

\section{Correlations between improvement of trunk balance and ADL}

Improvements in K-MBI were not correlated with improvement of K-BBS, PASS, or the static sitting balance subscale of TIS (Fig. 5). By contrast, it was positively correlated with improvement in the total score, the dynamic sitting balance subscale and the coordination subscale of TIS.

\section{DISCUSSION}

The results of the current randomized study indicated that trunk balance significantly improved in response to tNMES, as well as the CMS program in stroke patients, based on trunk balance scales. Furthermore, there were more significant improvements in trunk balance when the CMS program was applied in combination with tNMES therapy. The changes of K-MBI in the combination group were slightly higher than those in the other groups, but the difference was not statistically significant.

K-BBS, PASS, and TIS are well-known criteria that can be used to evaluate trunk balance [14-17]. The current analyses indicated significant improvements in K-BBS and the dynamic sitting balance of TIS when CMS was applied in combination with tNMES, but not for the other categories of TIS, such as in static sitting balance or trunk coordination. TIS is composed of 3 items to assess static sitting balance (scored as 0-7) evaluating trunk performance in anterior-posterior plane, 10 items to assess dynamic sitting balance (scored as $0-10$ ) evaluating lateral flexion of the trunk, and 4 items to assess trunk coordination (scored as 0-6) evaluating rotation of the trunk. In our opinion, only the dynamic sitting balance of TIS showed significant difference, because the mean score on the static sitting balance subscale for patients before treatment was nearly submaximal, i.e., $>4$ out of a maximum 7 points, whereas $<4$ out of a maximum 10 points in the dynamic sitting balance subscale and $<2$ out of a maximum 6 points in the coordination subscale of TIS. Therefore, the static sitting balance subscale might have shown a ceiling effect. Additionally, there is evidence of a hierarchy in the subscales of the TIS, as the dynamic sitting balance subscales seem to be more difficult than 


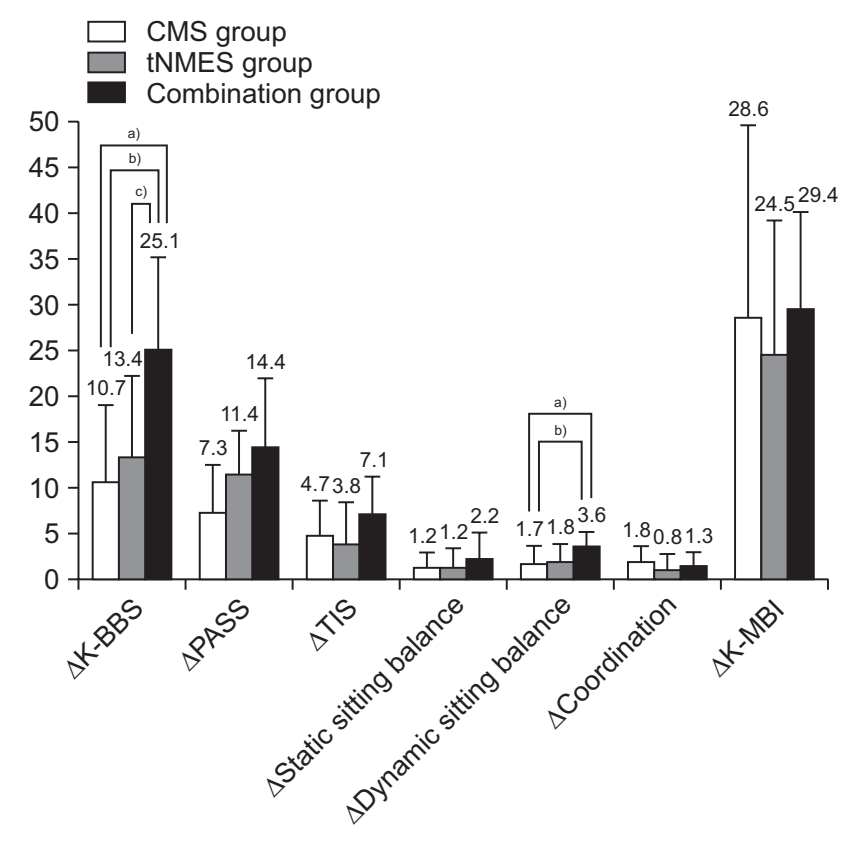

Fig. 4. The comparison of improvements in K-BBS, PASS, TIS, and K-MBI among 3 groups. CMS group, core muscle strengthening group; tNMES group, trunk neuromuscular electrical stimulation group; Combination group, core muscle strengthening and trunk neuromuscular electrical stimulation group; K-BBS, Korean version of Berg Balance Scale; PASS, total score of postural assessment scale for stroke patients; TIS, Trunk Impairment Scale; KMBI, Korean version of Modified Barthel Index. ${ }^{a)} \mathrm{p}<0.05$ by the Kruskal-Wallis test comparing CMS, tNMES, and combination groups, ${ }^{\text {b) }} \mathrm{p}<0.025$ by the Mann-Whitney Utest with the Bonferroni correction to compare CMS and combination group, ${ }^{c} \mathrm{p}<0.025$ by the Mann-Whitney Utest with the Bonferroni correction to compare tNMES and combination group.

the static sitting balance subscales, and the coordination subscales seem to be more difficult than the dynamic sitting balance subscales [18]. It is likely that there was insufficient intervention time for a significant difference in the coordination subscale, and extra training might be needed for it. Our results are consistent with those of a previous study [19], which showed that trunk exercises result in improvements only in the dynamic sitting balance subscale of the TIS. Van Nes et al. [20] reported a strong association of lateral balance control with BBS, indicating the need for lateral trunk control rehabilitation as a primary target.

Another study suggested a clear relationship between trunk performance and measurements of balance, gait, and functional ability after a stroke [21]. Dickstein et al. [8] showed that impairment of postural adjustments that are required for stability in the sitting position and functional deficits showed a positive relationship in stroke patients. In the present report, improvement of K-MBI, which represents functional status showed an association with improvement of the total score, and the dynamic sitting balance and coordination subscales of the TIS. Therefore, improved trunk balance could result in better functional recovery after a stroke, especially when improving the lateral flexion and rotation of the upper and lower parts of the trunk. As the combination group showed greater improvement in trunk performances than the CMS or tNMES groups, greater improvement of K-MBI was also expected in the combination group. However, our data showed no significant improvements in K-MBI in the combination group, possibly due to the short follow-up period.

Balance is a complex process that requires interactions among the vestibular, visual, proprioceptive, musculoskeletal, and cognitive systems. Both the trunk and limb muscles are involved in the coordination and regulation of automatic postural responses [6]. The muscles around the abdominal, lumbar, and pelvic regions play important roles in providing stability while maintaining balance [22]. The 'core' has been described as a box with the abdominals in the front, the paraspinals and gluteals in the back, the diaphragm as the roof, and the pelvic floor and hip girdle musculature as the bottom [23]; all of these muscles play an important role in maintaining stability [22]. Although each of the muscles related to core stability have individual roles, they function together via cooperative contractions to establish core stability. For example, the cooperative contractions of the transverses abdominis and multifidus muscles improve the stability of each component when the spine is in motion or even in a neutral position [24]. We provided verbal cues when the CMS program was performed to induce individual contractions in each muscle. This CMS program included simultaneous muscle strengthening and muscle control. Verheyden et al. [19] conducted core exercises for 30 minutes, 4 times per week over a period of 5 weeks, and detected an increase in the ability to maintain a sitting position. Additionally, Yu and Park [12] reported that core stability enhancing exercises are effective in improving muscle activities of the lower trunk, which are affected by 
(A)

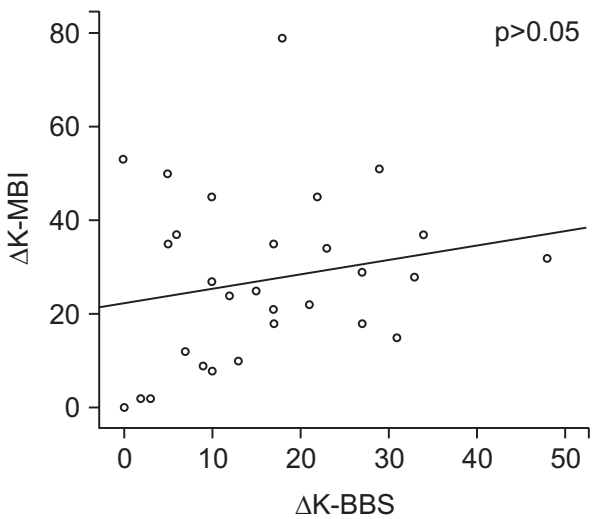

(D)

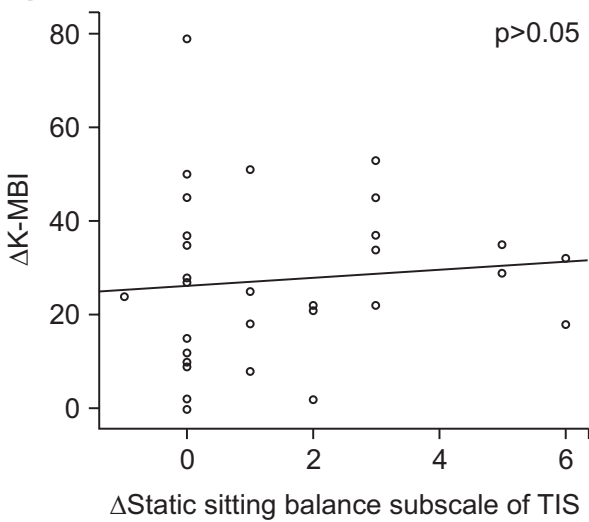

(B)

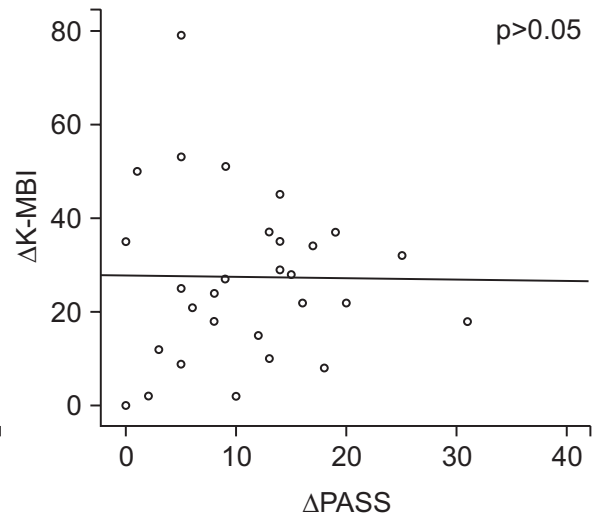

(E)

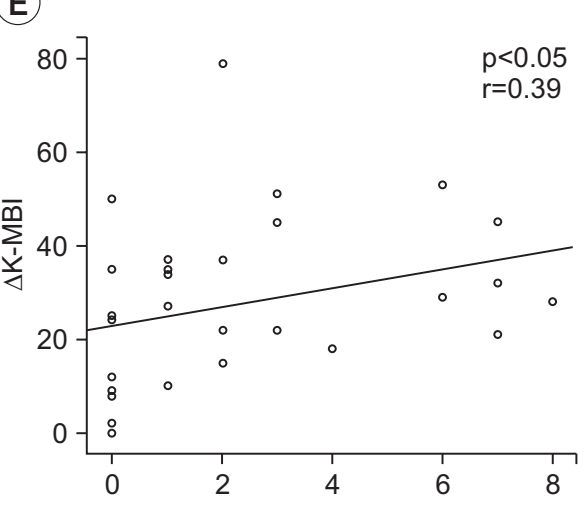

$\Delta$ Dynamic sitting balance subscale of TIS
(C)

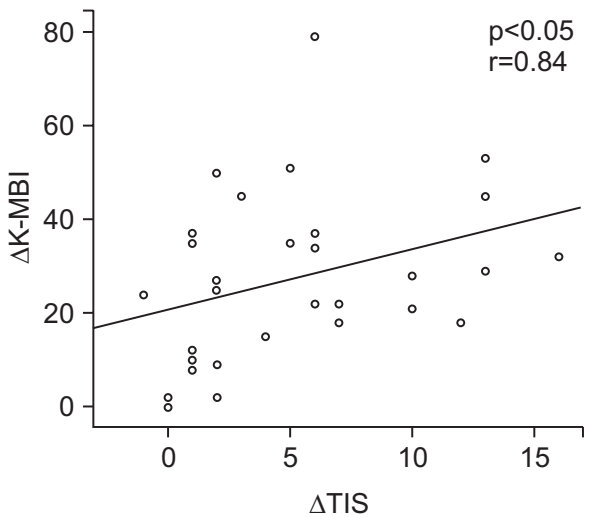

(F)

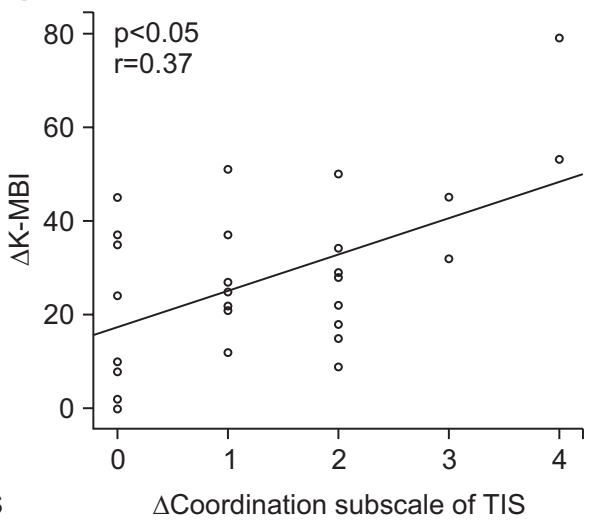

Fig. 5. Correlation between $\Delta \mathrm{K}$-MBI and (A) $\Delta \mathrm{K}$-BBS, (B) $\Delta$ PASS, (C) $\Delta \mathrm{TIS}$, (D) $\Delta$ static sitting balance subscale of TIS, (E) $\Delta$ dynamic sitting balance subscale of TIS, and (F) $\Delta$ coordination subscale of TIS. K-BBS, Korean version of Berg Balance Scale; PASS, total score of postural assessment scale for stroke patients; TIS, Trunk Impairment Scale; K-MBI, Korean version of Modified Barthel Index.

stroke.

According to Tanaka et al. [25], isometric trunk muscle strengths for flexion and extension in hemiplegic patients were $88 \%$ and $64 \%$, as compared to the healthy controls, and isometric and isokinetic muscle strengths for extension were weaker than that of the flexion. Therefore, we applied tNMES to paraspinal muscles to strengthen trunk extension in our study. Paraspinal muscles are surface muscles that are located among the extensors iliocostalis and longissimus. These muscles assist the multifidus muscle, which maintains the spine in a neutral position during the various curvatures caused by movements of the abdominal muscles [12]. Several previous studies have shown that performing somatosensory stimulation using neuromuscular electrical stimulation (NMES) in stroke patients can increase cortical excitability, as measured by functional magnetic resonance imaging $[26,27]$, and result in improvement of strength [26]. Repetitive movements caused by NMES induce long-term plasticity in motor maps that involve the development of new motor skills [28]. We used the same method of stimulating trunk muscles as the one used in the a previous study [13].

There are several possible explanations for trunk muscle weakness in hemiplegic patients. As mentioned before, because the motor cortex of both hemispheres supply nerves to the trunk, an upper motor lesion can cause bilateral trunk weakness [6]. Alternatively, patients who experience a cerebrovascular accident can lack selective movement control resulting in changes of the order of muscle movement. Therefore, these patients move in an unusual pattern, which results in inefficient energy usage and a malfunctioning movement pattern [12]. Trunk muscle weakness caused by disuse represents another possible reason [6]. 
There are some limitations to the generalization of the results of our study. First, there was no control group without intervention, so it is difficult to exclude effects of the natural recovery process of stroke. Second, all patients were aware of the treatment allocation scheme, and this could have introduced bias into the study. Third, we did not perform a long-term follow-up assessment; hence, future studies are required to evaluate long-term effects. Fourth, we did not use surface electromyography to assess trunk muscle activity as a measure of trunk strength, which could have improved our study. Fifth, only a small number of patients from a single geographical location were enrolled. Further studies including a larger patient cohort will be needed to fully verify our results.

The current results show that there is an additive effect of CMS and tNMES on the recovery of trunk balance, as compared to CMS or tNMES alone in patients with acute or subacute stroke who have poor sitting balance. In the current study, we aimed to emphasize the importance of treating the trunk, and introduce a combination therapy of CMS and tNMES together for improving trunk balance in stroke patients in a daily rehabilitation setting. Additional exercises performed on the trunk would allow patients to acquire better trunk balance, and this would lead to more functional improvements.

The prompt assessment and management of trunk control after a stroke are highly recommended, and the simultaneous application of CMS and tNMES should be considered when designing a rehabilitation program to improve trunk balance in stroke patients. Reestablishing trunk muscle function would improve the stability of stroke patients, and make it easier to re-educate limb muscles and gait patterns. To extend the findings of this study, chronic stroke patients should also be examined to rule out the effect of natural recovery from stroke.

\section{CONFLICT OF INTEREST}

No potential conflict of interest relevant to this article was reported.

\section{REFERENCES}

1. Warlow CP, van Gijn J, Dennis MS, Wardlaw JM, Bamford JM, Hankey GJ, et al. Stroke: practical manage- ment. 3rd ed. Malden: Blackwell Publishing; 2008.

2. Merkert J, Butz S, Nieczaj R, Steinhagen-Thiessen E, Eckardt R. Combined whole body vibration and balance training using Vibrosphere: improvement of trunk stability, muscle tone, and postural control in stroke patients during early geriatric rehabilitation. Z Gerontol Geriatr 2011;44:256-61.

3. Perlmutter S, Lin F, Makhsous M. Quantitative analysis of static sitting posture in chronic stroke. Gait Posture 2010;32:53-6.

4. Carr LJ, Harrison LM, Stephens JA. Evidence for bilateral innervation of certain homologous motoneurone pools in man. J Physiol 1994;475:217-27.

5. Yoo SD, Jeong YS, Kim DH, Lee MA, Noh SG, Shin YW, et al. The efficacy of core strengthening on the trunk balance in patients with subacute stroke. J Korean Acad Rehabil Med 2010;34:677-82.

6. Karatas M, Cetin N, Bayramoglu M, Dilek A. Trunk muscle strength in relation to balance and functional disability in unihemispheric stroke patients. Am J Phys Med Rehabil 2004;83:81-7.

7. Slijper H, Latash ML, Rao N, Aruin AS. Task-specific modulation of anticipatory postural adjustments in individuals with hemiparesis. Clin Neurophysiol 2002;113:642-55.

8. Dickstein R, Shefi S, Marcovitz E, Villa Y. Anticipatory postural adjustment in selected trunk muscles in post stroke hemiparetic patients. Arch Phys Med Rehabil 2004;85:261-7.

9. Hsieh CL, Sheu CF, Hsueh IP, Wang CH. Trunk control as an early predictor of comprehensive activities of daily living function in stroke patients. Stroke 2002;33:2626-30.

10. Moreland J, Thomson MA. Efficacy of electromyographic biofeedback compared with conventional physical therapy for upper-extremity function in patients following stroke: a research overview and metaanalysis. Phys Ther 1994;74:534-47.

11. van der Lee JH, Snels IA, Beckerman H, Lankhorst GJ, Wagenaar RC, Bouter LM. Exercise therapy for arm function in stroke patients: a systematic review of randomized controlled trials. Clin Rehabil 2001;15:20-31.

12. Yu SH, Park SD. The effects of core stability strength exercise on muscle activity and trunk impairment scale in stroke patients. J Exerc Rehabil 2013;9:362-7.

13. Kim YM, Chun MH, Kang SH, Ahn WH. The effect of 
neuromuscular electrical stimulation on trunk control in hemiparetic stroke patients. J Korean Acad Rehabil Med 2009;33:265-70.

14. Blum L, Korner-Bitensky N. Usefulness of the Berg Balance Scale in stroke rehabilitation: a systematic review. Phys Ther 2008;88:559-66.

15. Benaim C, Perennou DA, Villy J, Rousseaux M, Pelissier JY. Validation of a standardized assessment of postural control in stroke patients: the Postural Assessment Scale for Stroke Patients (PASS). Stroke 1999; 30:1862-8.

16. Verheyden G, Nieuwboer A, Mertin J, Preger R, Kiekens C, De Weerdt W. The Trunk Impairment Scale: a new tool to measure motor impairment of the trunk after stroke. Clin Rehabil 2004;18:326-34.

17. Jung HY, Park JH, Shim JJ, Kim MJ, Hwang MR, Kim SH. Reliability test of Korean version of Berg Balance Scale. J Korean Acad Rehabil Med 2006;30:611-8.

18. Verheyden G, Nieuwboer A, Feys H, Thijs V, Vaes K, De Weerdt W. Discriminant ability of the Trunk Impairment Scale: a comparison between stroke patients and healthy individuals. Disabil Rehabil 2005;27:10238.

19. Verheyden G, Vereeck L, Truijen S, Troch M, Lafosse C, Saeys W, et al. Additional exercises improve trunk performance after stroke: a pilot randomized controlled trial. Neurorehabil Neural Repair 2009;23:2816.

20. Van Nes IJ, Nienhuis B, Latour H, Geurts AC. Posturo- graphic assessment of sitting balance recovery in the subacute phase of stroke. Gait Posture 2008;28:507-12.

21. Verheyden G, Vereeck L, Truijen S, Troch M, Herregodts I, Lafosse $\mathrm{C}$, et al. Trunk performance after stroke and the relationship with balance, gait and functional ability. Clin Rehabil 2006;20:451-8.

22. Hodges PW. Core stability exercise in chronic low back pain. Orthop Clin North Am 2003;34:245-54.

23. Richardson CA, Jull GA, Hodges PW, Hides JA. Therapeutic exercise for spinal segmental stabilization in low back pain: scientific basis and clinical approach. Edinburgh: Churchill Livingstone; 1999.

24. Porterfield JA, DeRosa C. Mechanical low back pain: perspectives in functional anatomy. 2nd ed. Philadelphia: W. B. Saunders; 1998.

25. Tanaka S, Hachisuka K, Ogata H. Muscle strength of trunk flexion-extension in post-stroke hemiplegic patients. Am J Phys Med Rehabil 1998;77:288-90.

26. Kimberley TJ, Lewis SM, Auerbach EJ, Dorsey LL, Lojovich JM, Carey JR. Electrical stimulation driving functional improvements and cortical changes in subjects with stroke. Exp Brain Res 2004;154:450-60.

27. Wu CW, van Gelderen P, Hanakawa T, Yaseen Z, Cohen LG. Enduring representational plasticity after somatosensory stimulation. Neuroimage 2005;27:87284.

28. Sheffler LR, Chae J. Neuromuscular electrical stimulation in neurorehabilitation. Muscle Nerve 2007; 35:562-90. 